\title{
MRI role in the periprosthetic lymphoma screening: a case report
}

\section{La Forgia ${ }^{\#}$, A. Armenio², D. Cutrignelli², V. De Santis², A. Fanizzi ${ }^{*}$, A. Maiorella' ${ }^{2}$ A. Scattone ${ }^{*}$, C.M. Ressa ${ }^{2}$}

\author{
${ }^{1}$ I.R.C.C.S. Istituto Tumori “Giovanni Paolo II", Diagnosis and Imaging Therapy Department, Bari, Italy \\ 2I.R.C.C.S. Istituto Tumori “Giovanni Paolo II", Plastic Surgery Department, Bari, Italy \\ 3I.R.C.C.S. Istituto Tumori “Giovanni Paolo II”, Department of Pathology, Bari, Italy
}

\section{- Case report}

\author{
*Corresponding author: \\ Annarita Fanizzi, Ph.D., \\ E-mail: \\ annarita.fanizzi.af@gmail.com \\ Revised: July 2020 \\ Accepted: August 2020 \\ Int. J. Radiat. Res., October 2021; \\ 19(4): 1055-1058 \\ DOI: $10.29242 /$ ijrr.19.4.1055
}

\#These authors contributed equally to this work.

\section{ABSTRACT}

Anaplastic large T-cell lymphoma (BI-ALCL) is a rare primitive lymphoma found particularly in women with textured breast implant prostheses, which has been arousing interest in recent years due to its potentially high social impact. The difficult diagnosis together with the increasingly high number of prosthetic implants worldwide has led to a hypothesize of an underestimation of its real impact in prosthesis-bearing women. The clinical onset usually occurs with a cold seroma and disease confined to the prosthetic capsule while the more advanced stages may involve 1 or more lymph nodes or, rarely, distant metastases. Early diagnosis is essential as the disease has a favorable prognosis in over $90 \%$ of cases when detected in the early stages while it often has a poor prognosis in the advanced ones: despite this, the radiological signs of early disease are still non-specific. This report describes a case of BI-ALCL confined to the capsule which was studied using all diagnostic techniques in order to highlight its early radiological signs.

Keywords: Anaplastic large-cell lymphoma, breast magnetic resonance, breast cancer, radiomic analysis, computer-aided diagnosis.

\section{INTRODUCTION}

A 62 year old woman, with left breast cancer and a positive family history of breast cancer (mother), underwent a skin sparing mastectomy in 2009 with the positioning of an expander (Allergan/inamed $350 \mathrm{~cm}^{3}$ ) and subsequent reconstruction using a textured anatomical Allergan prosthesis $320 \mathrm{gr}$ located in a submuscular pocket. In 2014, owing to capsular contracture, she underwent a new operation to replace the old prothesis with a new textured Allergan prosthesis. In November 2019 she again came to our notice due to a progressive swelling in the left breast resulting in evident breast asymmetry (figure 1). A mammography showed a double contour surrounding the prosthesis (figure 2) compatible with periprosthetic effusion, while an ultrasound examination (US) confirmed an abundant periprosthetic seroma from which $300 \mathrm{ml}$ of turbid serous fluid was extracted using an ultrasound-guided needle aspiration for cytological analysis. Although axillary, supraclavicular and inguinal lymphadenopaties were not detected, though a breast Magnetic Resonance (MRI) confirmed a large periprosthetic collection of exudate which was enveloping and compressing the prosthesis. Some minute solid aggregates, attributable to fibrin deposits and amorphous material were found in the posterior area between the capsule and the prosthesis (figure 3 ).

The equipment used were the GE Senographe Essential mammography, Philips Affiniti 70 ultrasound, breast MRI was performed with 1.5 Tesla device (Achieva ${ }^{\circ}$, Philips Medical Systems, Best, The Netherlands) .

The cytological analysis of the collected fluid was performed on smears slides and cell-blocks. 
They showed amorphous acidophilic material incorporating lymphocytes apoptotic cells and and foamy histiocytes as well as a number of large-sized atypical cells with irregularly-shaped nuclei and an abundance of clear and often vacuolated cytoplasm. The atypical cells showed CD30 and T-cell markers (CD4) which accounted for $90 \%$ of the total cellularity compatible with the BI-ALCL diagnosis (figure 4a-b).

Total body CT and PET did not reveal signs of any secondary disease.

The diagnosis was confirmed by histological

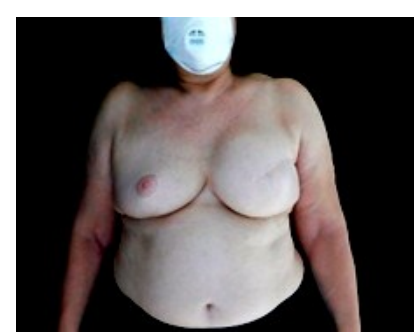

Figure 1. Clinical examination: breast asymmetry due to volumetric increase in the left breast.

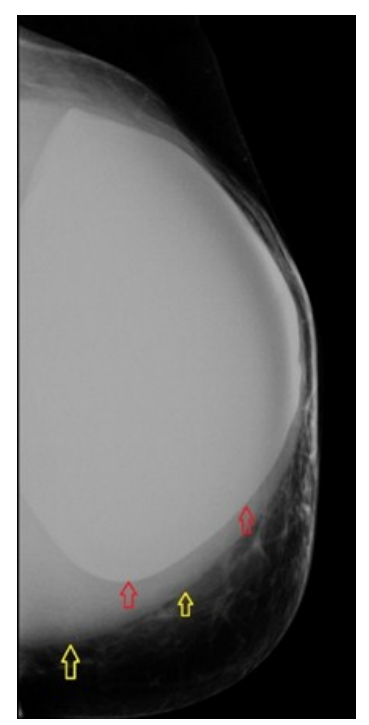

Figure 2. Mammography: double contour surrounding the prosthesis (red arrows) which reveals periprosthetic seroma (yellow arrows).

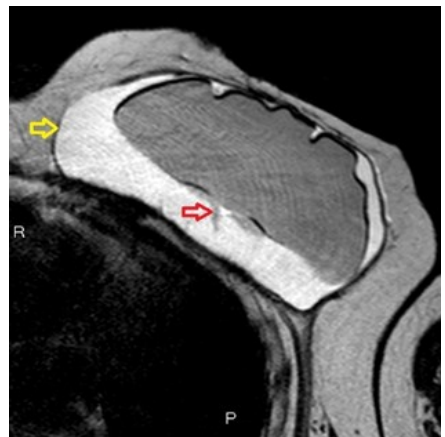

Figure 3. MRI: large fluid collection (yellow arrow) between capsule and prosthesis where some fibrin deposits are detectable in the posterior surface of the implant (red arrow).

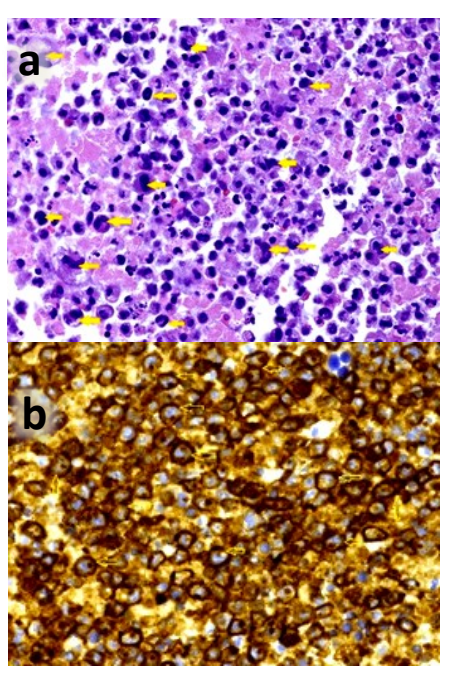

Figure 4a. Cytology: large cells with pleomorphic nuclei (yellow arrows) detected in a cytocentrifuge; they are admixed with fibrin and some inflammatory elements (hematoxylin and eosin, x40). b. Cytology: strong and diffuse presence of the CD30 molecule (yellow arrows) in neoplastic cells (anti-CD30 immunostaining, $\times 40$ ). examination following an en bloc capsulectomy with the implant removal (figure 5a-b): the malignant cells were confined within the capsule wall, in the intracapsular fluid and in a layer of fibrinous tissue adhering to the inner surface of the capsule where necrotic debris was found. There was evidence of superficial infiltration of the capsule itself. (figure 6a-b).

The patient did not undergo chemotherapy or radiotherapy due to the local confinement of the disease.

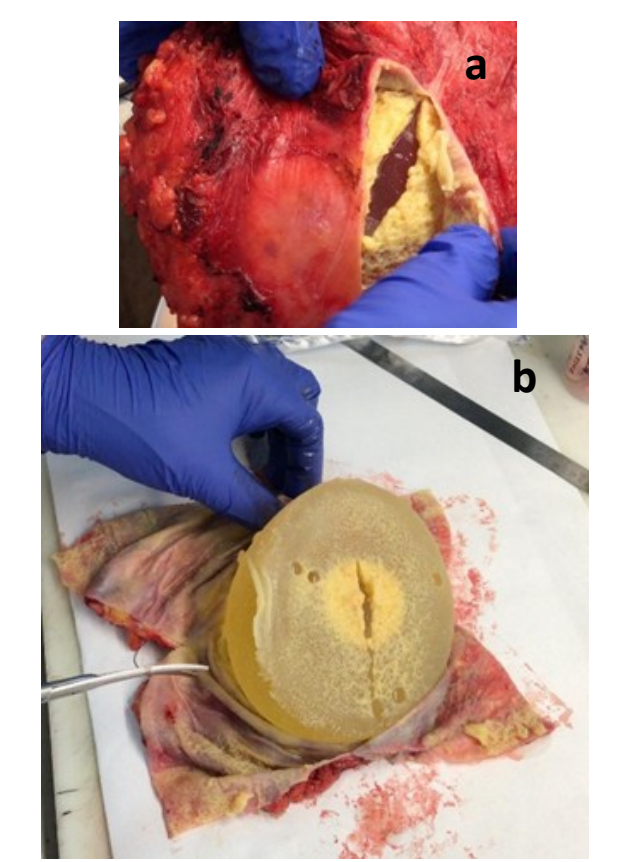

Figure 5a. Surgery: details of the capsule opening clearly showing the inner aspect of the capsule and the fibrin layer. b. Post-surgery: details of the inner aspect of the capsule once opened. The yellowish fibrin tissue is clearly visible adhering to the lower part of the implant. 

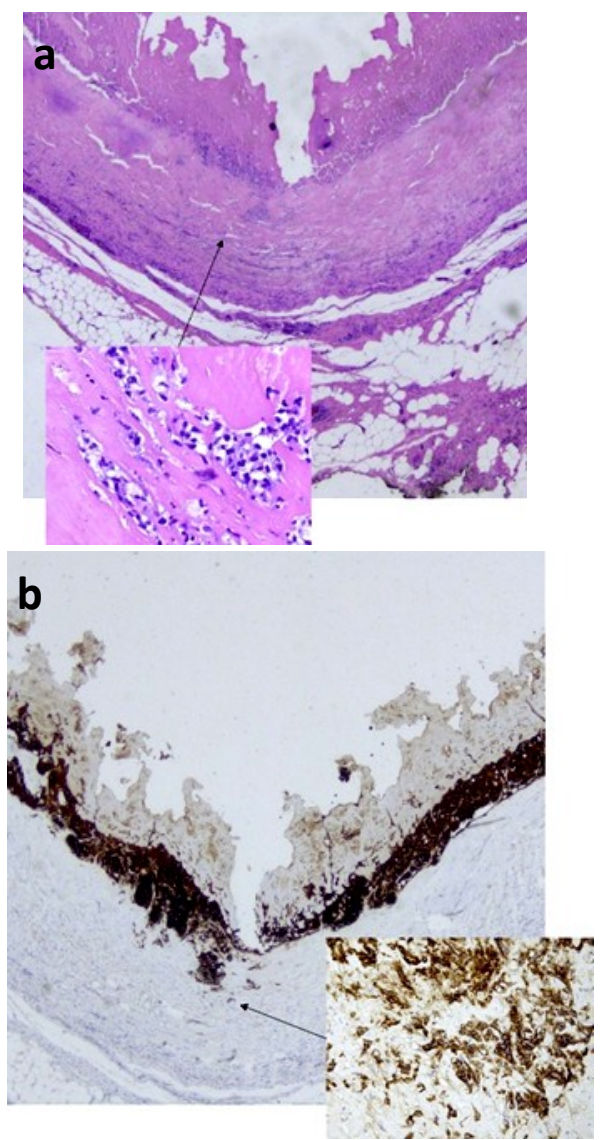

Figure 6a. Definitive histology (on capsulectomy): Neoplastic cells surrounded by a clear halo are contained inside a fibrin layer and infiltrate the inner part of the capsule; the rest of the capsule shows scattered inflammatory elements (hematoxylin and eosin, x2.5 was photographed at $25 \mathrm{x}$ original magnification); black arrow shows sheets of lymhoma cells into the capsule. Inset: malignant cells into the area of invasion (hematoxylin and eosin photographed at 200x original magnification). b. Anti CD30 stain of atypical cells lining the fibrous capsule; black arrow highlights infiltration of neoplastic cells into the caspsule (anti-CD30 immunostaining was photographed at $25 x$ original magnification). Inset: malignant cells positive with anti-CD30 immunostaining into the area of invasion (anti-CD30 immunostaining photographed at $200 x$ original magnification).

\section{DISCUSSION}

Anaplastic large cell periprosthetic lymphoma (BI-ALCL), first described in literature in 1997 by Keech and Creech (1,2), is a rare disease which constitutes $0.04-0.5 \%$ of all malignancies $(2,3,4)$.

The progressive increase in prosthetic implants worldwide, with more than 1.5 million new procedures a year (5-7) and the non-specific symptoms and radiological signs of the disease have led to a growing need for detecting the disease in its early stages.

The case report highlights that cytological evaluation and CD30 immunostaining of late peri-implant breast seroma allow early recognition of BI-ALCL and that radiological signs are the most effective tool for screeing patients for BI-ALCL.

The pathogenetic mechanism of BI-ALCL originates as a chronic inflammation caused by the prosthesis, expecially when textured, and its degradation products (8-13): one of which is fibrin, visible in MRI as reported in a recent retrospective study (14). This, together with cell necrosis products, are confirmed as an early radiological indicator of the disease in the present study as well: these signs appear specific to this disease and should not be included in differential diagnosis with prosthetic ruptures.

US, used in clinical practice alone or with other support systems $(15,16)$, can be used in the diagnosis of BI-ALCL as a guide for cytological sampling on seroma but, unilike the MRI, fails to provide a multiparametric vision.

Periprosthetic seroma, which often represents the first stage of the disease, is a non specific sign and as such the diagnosis can often be delayed: for this reason, the identification of early radiological signs is crucial for the correct diagnostic classification and to favour the complete remission of the disease in more than $90 \%$ of cases after capsulectomy alone; on the contrary, a more aggressive outcome is generally observed in advanced stages $(17,18)$.

A limitation could be constituted by the detection of fibrin inside the seroma: in this case an important aid could come from the CAD systems which, if correctly used show a performances superior to that of the operator in the recognition and characterization of small breast lesions (19-26) or by fusion-imaging US/ MRI (16).

This would suggest a greater use of MRI in the monitoring of periprosthetic seromas with undefined etiopathogenesis. In addition, the integration of CD30 immunostaining cytological analysis represents the most effective diagnostic 
algorithm for the diagnosis in daily practice ${ }^{(27) .}$

\section{ACKNOWLEDGMENT}

This research did not receive any specific grant from funding agencies in the public, commercial, no-profit sectors. The authors thank Grazia Giannone, Rosanna Digirolamo, Michele Siciliano and Donato Loisi for technical assistance.

\section{Conflicts of interest: Declared none.}

\section{REFERENCES}

1. Cohen PL and Brooks J (1991) Lymphomas of the breast: a clinicopathologic and immunohistochemical study of primary and secondary cases. Cancer, 67: 1359-69.

2. Keech JA (1997) Anaplastic T-cell lymphoma in proximity to a saline-filled breast implant. Plast Reconstr Surg, 1997100: 554-5.

3. Thompson PA and Prince HM (2013) Breast implantassociated anaplastic large cell lymphoma: a systematic review of the literature and mini-meta analysis. Curr $\mathrm{He}$ matol Malig Rep, 8: 196-210.

4. TGA, Breast implants and Anaplastic Large Cell Lymphoma, Therapeutic Goods Administration. https:// www.tga.gov.au/alert/breast-implants-and-anaplasticlargecell-lymphoma.

5. ISAPS, The International study on aesthetic / cosmetic procedures performed in 2016, accessed December 2017.

6. US Food and Drugs Administration, et al. (2011) Anaplastic large cell lymphoma (ALCL) in women with breast implants: Preliminary FDA findings and analyses. Center for Devices and Radiological Health Silver Spring, MD (2011).

7. Surgeons ASoP, Report of the 2010 Plastic Surgery Statistics (2011).

8. Doren EL, Miranda RN, Selber JC et al. (2017) US epidemiology of breast implant-associated anaplastic large cell lymphoma. Plast Reconstr Surg, 139(5): 1042-1050.

9. De Boer M, Van Leeuwen FE, Hauptmann M, et al. (2018) Breast implants and the risk of anaplastic large-cell lymphoma in the breast. JAMA Oncol, 4(3): 335-341.

10. Clemens MW, Nava MB, Rocco N, et al. (2017) Understanding rare adverse sequelae of breast implants: anaplastic large-cell lymphoma, late seromas, and double capsules. Gland Surg, 6: 169-84.

11. Xu J and Wei S (2014) Breast implant-askksociated anaplastic large cell lymphoma: Review of a distinct clinicopathologic entity. Arch Pathol Lab Med, 138(6): 842-846.

12. Hart AM, Lechowicz MJ, Peters KK, et al. (2014) Breast implant-associated anaplastic large cell lymphoma: report of 2 cases and review of the literature. Aesthet Surg J, 34
(6): 884-894.

13. Berlin E, Singh K, Mills C, et al. (2018) Breast implantassociated anaplastic large cell lymphoma: Case report and review of the literature. Case Rep Hematol, 2414278.

14. La Forgia D, Moschetta M, Fausto A, et al. (2019) Anaplastic large-cell periprosthetic lymphoma of the breast: could fibrin be an early radiological indicator of the presence of disease? JBUON, 24(5): 1889-1897.

15. Tagliafico AS, Mariscotti G, Valdora F, et al. (2018) A prospective comparative trial of adjunct screening with tomosynthesis or ultrasound in women with mammographynegative dense breasts (ASTOUND-2). European Journal of Cancer, 104: 39-46.

16. Fausto A, Bernini M, La Forgia D, et al. (2019) Six-year prospective evaluation of second-look US with volume navigation for MRI-detected additional breast lesions. European Radiology, 29(4): 1799-1808.

17. Miranda RN, Aladily TN, Prince HM, et al. (2014) Breast implant-associated anaplastic large cell lymphoma: longterm follow-up of 60 patients. J Clin Oncol, 32(2): 114-120.

18. Alobeid B, Sevilla DW, El-Tamer MB, et al. (2009) Aggressive presentation of breast implant-associated ALK-1 negative anaplastic large cell lymphoma with bilateral axillary lymph node involvement. Leuk Lymphoma, 50(5): 831833.

19. Fanizzi A, Basile TMA, Losurdo L, et al. (2017) Hough Transform for Clustered Microcalcifications Detection in FullField Digital Mammograms. Proceedings of SPIE - The International Society for Optical Engineering, 10396: 1039616.

20. Losurdo L, Fanizzi A, Basile TMA, et al. (2018) A combined approach of multiscale texture analysis and interest point/ corner detectors for microcalcifications diagnosis. Lecture Notes in Computer Science, 10813 LNBI: 302-313.

21. Losurdo L, Basile TMA, Fanizzi A, et al. (2018) A gradientbased approach for breast DCE-MRI analysis. BioMed Research International, 9032408.

22. Basile TMA, Fanizzi A, Losurdo L, et al. (2019) Microcalcification detection in full-field digital mammograms: A fully automated computer-aided system. Physica Medica, 64: 1 $-9$.

23. Losurdo L, Fanizzi A, Basile TMA, et al. (2019) Radiomics analysis on contrast-enhanced spectral mammography images for breast cancer diagnosis: A pilot study. Entropy, 21(11): 1110.

24. Fanizzi A, Basile TMA, Losurdo L (2019) Ensemble discrete wavelet transform and gray-level co-occurrence matrix for microcalcification cluster classification in digital mammography. Applied Sciences, 9(24): 5388.

25. Fanizzi A, Losurdo L, Basile TMA, et al. (2019) Fully automated support system for diagnosis of breast cancer in contrast-enhanced spectral mammography images. J Clin Med 8: 891.

26. Fanizzi A, Basile TMA., Losurdo L, et al. (2020) A machine learning approach on multiscale texture analysis for breast microcalcication diagnosis. BMC Bioinformatics, 21(2): 91.

27. Di Napoli A, Pepe G, Giarnieri E, et al. (2017) Cytological diagnostic features of late breast implant seromas: From reactive to anaplastic large cell lymphoma. PLoS One, 17: 12(7). 